\title{
Detection of luxS Gene in Serratia marcescens and rpoS Gene in Enterobacter cloacae Isolates Using PCR Reaction
}

\author{
Naba'a A. Muhammed ${ }^{1 *}$, Muthana B. Farhan ${ }^{1}$ and Zeyad A. Shabeeb ${ }^{2}$ \\ ${ }^{1}$ Department of Biology, Faculty of Education for Women, University of Anbar, Fallujah-Iraq. \\ ${ }^{2}$ Department of Microbiology, Faculty of Medicine, University of Al-Mustansiriyah, Baghdad-Iraq. \\ *Corresponding Author: biologistnabaa@yahoo.com.
}

\begin{abstract}
luxS and rpoS genes were involved in the regulation of some biological processes, which interfere with virulence of Serratia marcescens and Enterobacter cloacae bacterial species sequentially. luxS have an important activity in Quorum Sensing (QS) processes, but rpoS interfere with tolerance and resistance processes toward stress conditions correlating with its virulence. The study aimed to detect rpoS presence in E. cloacae and luxS gene presence in $S$. marcescens separately isolated from 16 leukaemia patients (4 of S. marcescens and 12 of E. cloacae), who characterised with bacteraemia by using primers designed for this purpose by using PCR technique. The results showed that all of the bacterial isolates of $S$. marcescens contain luxS gene, while no one of E. cloacae isolates show positive results for rpoS gene. The study concluded that $S$. marcescens has high virulence as a result of luxS presence, which controls Quorum sensing and prodigiosin formation and affecting host immunity. Moreover, the negative results of rpoS gene can be explained according to two possibilities: the gene can be found in the isolates with a copy differ from the registered sequences of the $r p o S$ gene, or the gene did not present in those isolates with presence of alternatives offset its activities. [DOI: 10.22401/JUNS.21.1.18]
\end{abstract}

Keywords: rpoS gene, Enterobacter cloacae, luxS gene, Serratia marcescens.

\section{Introduction}

luxS gene correlates with Quorum sensing (QS) in many bacterial species [1]. The product of this gene expression is S-ribosylhomocysteine lyase (LuxS) enzyme, which is responsible for the production of Autoinducer -2 (Al-2) that involved in QS in many bacteria [2]. Previous studies indicated the importance of this gene in the regulation of QS pathway in $S$. marcescens, which expressed as a result to response for bacterial density [3]. So that it's involved in Al-2 dependent Quorum sensing [4], which include Pfs and LuxS enzymes that are responsible for the biological manufacturing of signal molecules, contribute to the virulence induction of human opportunistic pathogen Serratia [5]. LuxS enzyme plays a regulatory role in the metabolic activities of Serratia by regulation of production of some primary metabolites in addition to prodigiosin (one of the secondary metabolites) [6], which regulate by luxS gene in some $S$. marcescens strains. Moreover, any mutation in luxS leads to reduce its virulence factors like prodigiosin and haemolysin production [3, 7]. According to previous studies and what concluded by coulthurst et al [2] study, luxS interferes with prodigiosin production and regulate antibiotic production in Serratia spp. with a significant difference in its virulence factors. Many of cellular pathways developed in bacteria in order to tolerate severe stress conditions and one of those pathways was rpos gene responsible for RNA polymerase sigma $S$ (sigma 38) factor which interfere with virulence of many pathogens, particularly in stationary phase [8-12]. In E. cloacae, this gene considered one of regulatory genes through response to starvation, as well as its considered to be one of virulence factors such as regulation of hydrogen peroxide catalase II (HPII), which give it the ability to resist $\mathrm{H}_{2} \mathrm{O}_{2}$, In addition to regulation of toxins secretion from Serratia strains [10]. Since rpoS involved in the controlling of some virulence genes in pathogenic bacteria to enable those bacteria to resist stressed environments supplied by the host [13], which lead to the controlling of number of genes involved in cellular viability maintenance in stationary phase like oxidative stress, hyper-osmolality, starvation and 
reduced $\mathrm{PH}$ [13- 15]. In addition to that, the incidence of some changes in gene regions can lead to the formation of more virulent strains [11]. The study aimed to detect the presence of luxS gene in Serratia marcescens and rpoS gene in Enterobacter cloacae investigate its importance as virulence factors in the studied isolates.

\section{Materials And Methods}

\subsection{Bacterial Isolates}

The study performed on 4 bacterial isolates of $S$. marcescens and 12 bacterial isolates of E. cloacae from 33 bacterial isolates, which isolated from 60 blood samples of leukaemia patient from National Center of Haematology Researches and Therapy and from Baghdad Teaching Hospital from April to October (2016), who were suffered from some clinical signs and diagnosed with bacteraemia. The bacterial isolates characterised with Api-20E.

\subsection{Polymerase chain reaction (PCR) of luxS and rpoS}

\subsubsection{DNA Extraction}

Genomic DNA was extracted from the bacterial isolates using DNA extraction kit. (Promega, USA) and DNA purity was measured by NanoDrop device (Thermo scientific, USA), then the extracted DNA were subjected to electrophoresis to ensure its purity and integrity.

\subsubsection{Primers}

Specific primers were used for detection of rpoS and $\operatorname{luxS}$ genes, which designed with primer 3 program (as indicated in Table (1)) and supplied by Alpha DNA as lyophilized powder then dissolved with a specific volume of sterilized distilled water according to manufacturer recommendations to form stock solution for each primer with $(100 \mu \mathrm{M})$ concentration then its stored at $-20^{\circ} \mathrm{C}$.

Table (1)

Primers sequences and the product size for each one.

\begin{tabular}{|c|c|c|c|c|}
\hline Primer name & Gene ID* & Direction & Nucleotide sequence & Product size (bp) \\
\hline \multirow{2}{*}{$\operatorname{lux} S$} & \multirow{2}{*}{3278137} & $\mathrm{~F}^{* *}$ & TCATGGCATACCATCACGG $\rightarrow$ & \multirow{2}{*}{360} \\
\hline & & $\mathrm{R}$ & TCCAGAATGTGCTTGGCGAT $\leftarrow$ & \\
\hline \multirow{2}{*}{ rpos } & \multirow{2}{*}{9124132} & $\bar{F}$ & $\rightarrow$ AACGGTATTGGCCTGCCTTT & \multirow{2}{*}{354} \\
\hline & & $\mathrm{R}$ & $\leftarrow$ TGCCCCTGATGCGATAACTG & \\
\hline
\end{tabular}

* Gene ID obtained from GenBank/NCBI.

** F: Forward; R: Reverse.

\subsubsection{PCR Reaction mixture}

The PCR mixture purchased from BioNEER composed of Buffer solution, dNTPs and Taq DNA polymerase, which mixed like what described in Table (2). While the program used for PCR reaction of $\operatorname{lux} S$ and rpoS genes described in Table (3).

Table (2)

PCR mixture components and its volumes.

\begin{tabular}{|c||c||c|}
\hline Component & $\begin{array}{c}\text { The volume of } \\
\text { each component } \\
\text { in } \boldsymbol{\mu l}\end{array}$ & $\begin{array}{c}\text { The } \\
\text { concentration }\end{array}$ \\
\hline \hline Distilled water & 15 & - \\
\hline \hline Master mix & 5 & - \\
\hline \hline $\begin{array}{c}\text { Primer } \\
\text { forward }\end{array}$ & 1 & $1 \mu \mathrm{M}$ \\
\hline \hline Primer reverse & 1 & $1 \mu \mathrm{M}$ \\
\hline DNA template & 3 & $\begin{array}{c}\text { Depending on } \\
\text { the isolate* }\end{array}$ \\
\hline
\end{tabular}

*As indicated in Table (4). 
Table (3)

The program used in PCR Reaction of luxS and rpoS genes.

\begin{tabular}{|c|c|c|c|c|}
\hline \multirow{2}{*}{ Steps } & \multicolumn{2}{|c|}{ Temperature } & \multicolumn{2}{|c|}{ Time } \\
\hline & rpos & $\operatorname{luxS}$ & rpos & $\operatorname{luxS}$ \\
\hline Initial denaturation & $95^{\circ} \mathrm{C}$ & $95^{\circ} \mathrm{C}$ & 5 Min. & 5 Min. \\
\hline Denaturation & $95^{\circ} \mathrm{C}$ & $95^{\circ} \mathrm{C}$ & $45 \mathrm{Sec}$. & $45 \mathrm{Sec}$. \\
\hline Annealing & $57^{\circ} \mathrm{C}$ & $56^{\circ} \mathrm{C}$ & 45 Sec. & $40 \mathrm{Sec}$. \\
\hline \multicolumn{5}{|c|}{ Number of cycles: 35} \\
\hline Extension & $72^{\circ} \mathrm{C}$ & $72^{\circ} \mathrm{C}$ & 1 Min. & $25 \mathrm{Sec}$. \\
\hline Final Extension & $72^{\circ} \mathrm{C}$ & $72^{\circ} \mathrm{C}$ & 10 Min. & $10 \mathrm{Min}$. \\
\hline
\end{tabular}

\subsubsection{Gel electrophoresis}

Electrophoresis device was supplied with 1xTBE-buffer (Promega, USA) and Agarose gel plate prepared with $1 \%$ concentration of Agarose (BIOBASIC, Canada) with using of safe DNA loading dye (BIOland, USA) then $5 \mu l$ of PCR product of luxS or $r p o S$ and (100 bp) DNA ladder (BioNEER, Korea) was added to gel wells carefully before supplying of 50 volts of voltage for 5 minutes then 100 volts for 40 minutes. After that the gel plate was checked for the presence of bands using Desktop gel imager UV-transilluminator (SCOPE-21, Japan) under $312 \mathrm{~nm}$ UV light, then the molecular size of each band was detected according to the distances in comparison with DNA ladder.

Table (4)

The concentrations and purity of the genomic DNA of S. marsescens and E. cloacae bacterial isolates.

\begin{tabular}{||c||c||c||c||c||}
\hline Con(ng. I $\left.^{-1}\right)$ & 280/ 260 & Abs 280 & Abs 260 & Sample \\
\hline \hline 72.6 & 1.78 & 0.818 & 1.453 & E. cloacae \\
\hline 63.2 & 1.29 & 1.36 & 1.76 & E. cloacae \\
\hline \hline 186.5 & 1.77 & 2.105 & 3.729 & E. cloacae \\
\hline \hline 43.1 & 1.90 & 0.453 & 0.862 & E. cloacae \\
\hline \hline 87.4 & 1.60 & 1.091 & 1.749 & E. cloacae \\
\hline 80.3 & 1.68 & 1.56 & 2.63 & E. cloacae \\
\hline \hline 62.1 & 1.29 & 1.30 & 1.68 & E. cloacae \\
\hline \hline 85.7 & 2.25 & 0.432 & 0.976 & E. cloacae \\
\hline \hline 72.5 & 1.78 & 0.813 & 1.451 & E. cloacae \\
\hline 60.0 & 1.21 & 1.32 & 1.60 & E. cloacae \\
\hline \hline 86.4 & 1.60 & 1.08 & 1.73 & E. cloacae \\
\hline \hline 60.5 & 1.34 & 1.31 & 1.76 & E. cloacae \\
\hline \hline 80.1 & 1.58 & 1.58 & 2.50 & S.marcescens \\
\hline \hline 56.8 & 1.30 & 1.23 & 1.60 & S.marcescens \\
\hline \hline 48.3 & 1.89 & 0.83 & 1.57 & S.marcescens \\
\hline \hline 64.1 & 1.20 & 1.40 & 1.69 & S.marcescens \\
\hline
\end{tabular}




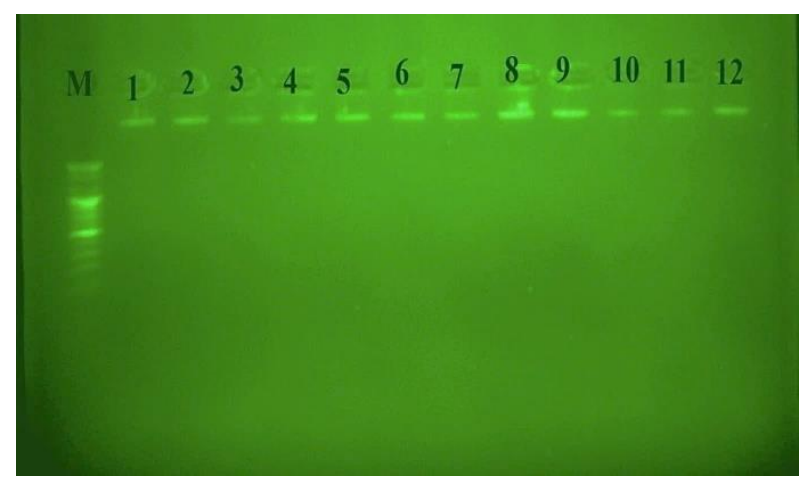

Fig.(2): Isolated DNA electrophoresis from Enterobacterclacae.

* M: Marker (100bp ladder).

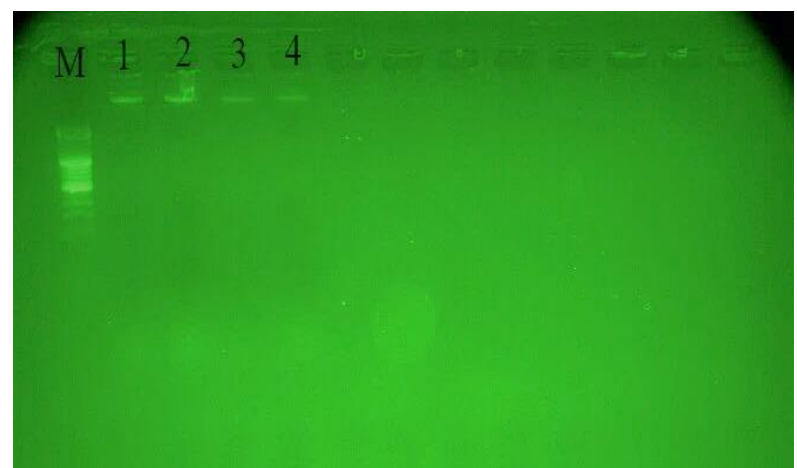

Fig.(3): Isolated DNA electrophoresis from Serratia marcescens.

* M: Marker (100bp ladder).

\subsection{Detection of luxS gene in Serratia marcescens:}

The results of $\operatorname{lux} S$ gene detection in $S$. marcescens showed that all of the isolated bacteria from leukaemic patients (4 isolates) who suffering from bacteraemia contain luxS gene as presented in Fig.(4).

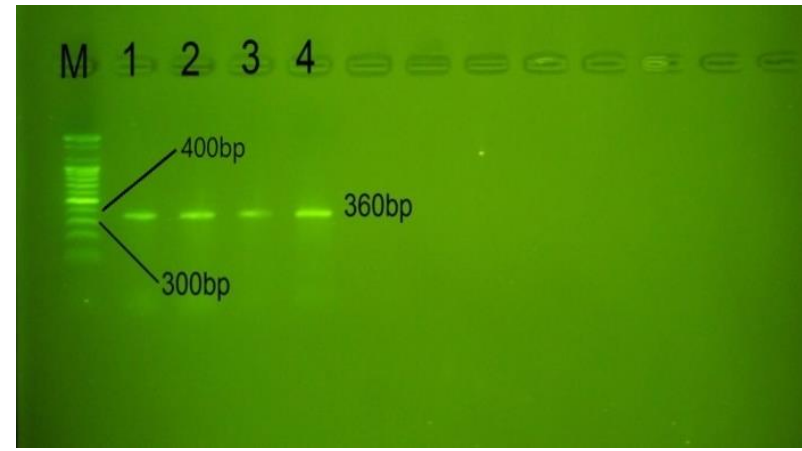

Fig.(4): PCR reaction product of luxS gene from Serratia marcescens.

* M: Marker (100bp ladder).

\subsection{Detection of rpos gene Enterobacter cloacae}

The results that obtained from PCR reaction for detection of $r p o S$ genes in $E$. cloacae, which are displayed in Fig.(5) elucidated that no one of E. cloacae isolates shows positive result to $r p o S$ gene

\section{Discussion}

The study aimed to detect the presence of luxS gene in $S$. marcescens isolated from leukaemia patients' blood samples and the study indicated that all of the isolates show positive results for the presence of this gene. This agrees with Coulthurst et al [2] study, which indicted that all of $S$. marcescens 274 and S. marcescens 39006 strains contain this gene, which gives it the ability to produce $\mathrm{Al}-2$ activity. Instead of the differences in the downstream region of this gene between $S$. marcescens strains, which caused by unknown reasons that lead to differences in gene regulatory control on different characteristics depending on the strains such as variations in prodigiosin production control between $S$. marcescens 274 and 39006. Moreover, luxS gene mutations in $S$. marcescens 274 only can lead to significant differences in its virulence [2]. Its role in virulence proved by many studies; since Sun et al [6] indicated that Al-2 production (one of QS inducers) was controlled by luxS gene transcription level in $S$. marcescens. and Coulthurst et al prove that S. marcescens 274 and 39006 strains produce significant amounts of Al-2 activity during activation of a chromosomal copy of the gene in both strains that prove the responsibility of the gene on Al-2 production in both strains. Nevertheless, it's decreased when bacteria enter into the stationary phase in S.marcescens. [2,6] In addition, another previous study revealed that the gene has an importance in Quorum Sensing regulation which plays an important regulatory role in the metabolic activities of S.marcescens by regulation of production of some primary metabolites as well as prodigiosin (from secondary metabolites), [6] this pigment regulated by QS and luxS in some strains of Serratia and considered to be an antibiotic and play an important role in virulence and its production controlled by complex process and depend on environmental processes, Since QS mechanism enable the bacteria to regulate gene expression as a response to bacterial density so that any mutation can lead to reduce prodigiosin and haemolysin production as well as virulence $[2,3,7]$ In spite of inability of our 
study to detect strain type of the isolates according to the obtained results, indeed; the strains colours were red or reddish pink, which demonstrates prodigiosin production like what appears since S. maecescens 274 and 39006 strains produce prodigiosin and all of these strains contain luxS gene, which improves the results obtained by PCR detection of the gene, but Coulthurst et al [2] prove luxS rule in the regulation of prodigiosin production in 274 strain but not in 39006 strain.

rpoS was an important factor in controlling of cells and assisting in bacterial persistence under stress condition and contribute to the virulence by many effects, Our study accomplished in the presence of $r p o S$ gene $E$. cloacae isolated from blood specimens from leukaemia patients. PCR results obtained by specific primers for $r p o S$, elucidated that no one of E. cloacae isolates appeared positive results. This result agree somewhat with Hussain and Alammar [8] study, which elucidated high possibility for the negative result of rpoS gene in E. cloacae because $31 \%$ only of Enterobacter isolates have rpoS gene (21 positive isolates from 75 isolates) which include $28 \%$ from E. cloacae and $56 \%$ of $E$. sakazakii. That study represented little probability for positive results and it accomplished in the same zone of our study, in addition to the differences in the gene appearance probability according to the differences in the studied numbers of isolates. Since that study included high significant number (84 isolates) in comparison to our study isolates' number (12 isolates), but no isolate of our study show positive result. Martinez-Garcia demonstrated that bacterial DNA, which fails to correlate with its specific primer of the gene in PCR reaction show a negative result, but it didn't indicate the absence of the gene also the isolates which show a negative result appeared to have less consensual sequences with $E$. coli $r p o S$ sequence as this gene considered to be widespread and conserve in Enterobacteriaceae so that the isolate which lacks the gene show less persistence under thermal and osmolality conditions. [10,12] Moreover, When Martinez- Garcia et al [10] create $E$. coli rpoS probe to detect the presence of the gene in Enterobacteriaceae including $E$. cloacae, he found that rpoS gene in E. cloacae similar to the gene in $E$. coli, since he studied the presence of the gene in E. cloacae CET 863 strain and he found that the identity percent was $80 \%$ between $E$. coli rpoS and $E$. cloacae rpos. While some Enterobacterceae members show negative results in both PCR and southern analysis in both Proteus Vulgaris and Providencia Stuartii. According to the absence of studies, which explain the reason for this negative result in addition to the inability to explain this reason, according to the results obtained from the study so that we can establish some hypotheses which must be studied later to explain this result. The hypotheses include the absence of the gene in the studied isolates strains according to inability to detect the strain from study results, in addition to presence of many strains follow E. cloacae, since the studied strains by Martinez-Garcia et al [10] and NavarroLiorens et al [16] show positive results for rpoS gene involving CETC 960 (free mutation wild-type strain), ATCC 13047 strain and RC10-RC1 strains. So that it can be subrogated by other factors to perform its functions in resistance to some stress conditions, for example: some E. coli strains which lack rpoS gene can adapt to stress conditions which include hyper osmolality by addition of mobile factor IS10 to ost-BA operon promoter [17], or the negative results can be resulted from differences in gene sequence in the studied isolates from the registered sequences which lead to inability to detect the gene, as well as can be resulted from the absence of the plasmid containing the gene since it's portable on pBEcl plasmid [10] or the mutations which can happened in the downstream region of the gene that effect on the ability of the primer to link to its complement sequence, for example, when Martinez- Garcia et al [10] induce addition mutation in rpoS gene in E. cloacae, which lead to replace of $r p o S$ wild-type gene with not working allele and the resulted strain named with E. cloacae E1. Few studies obtained on E. cloacae ability to produce virulence-related characteristics [8]. As it's considered to be an opportunistic pathogen responsible for Bacteremia [10] and it can enter in extraintestinal infections, because of the 
presence of virulence-related features like adhesion ability and invasion on Eukaryotic cells. While it has the ability to chelate the iron outside gastrointestinal tract so that it can live and spread inside the host and resist the environmental stresses like the effect accomplished by rpos [8].

\section{References}

[1] Joyner Jessica, Wanles David, SinGalliano Christopher D., Lipperin K., "Use of Quantitative Real-Time PCR for Direct Detection of Serratia marcescens in Marine and other Aquatic Environments", APPL. ENVIRON. MICROB. 80 (5), 1679-1683, 2014.

[2] Coulthurst Sarah J., Kurz C. Leopold, Salmond George P. C., "luxS Mutants of Serratia Defective in Autoinducer-2Dependent Quorum Sensing Show StrainDependent Impacts on Virulence and Production of Carbapenem and Prodigiosin", MICROBIOLOGY+. 150, 1901-1910, 2004.

[3] Martinez Katrina C., Teves Franco, Gandmadamba Ma, Reina Suztt B., "Sequence Analysis of Putative luxS gene Involved in Prodigiosin Biosynthesis from Philippine Local Stains of Serratia marcescens", INT. RES. J. BIOL. SCI. 2 (4), 13-19, 2013.

[4] Houdt Rob Van, Givskov Micheal, Michiels Chris W., "Quorum Sensing in Serratia", FEMS MICROBIOL REV. 37, 407-424, 2007.

[5] chauder Stephen, Shokat Kevan, Surette Michael G., Bassler Bonnie L., "The luxS Family of Bacterial Auto-inducers: Biosynthesis of A novel Quorum Sensing Signal Molecule", MOL BIOL+. 41 (2), 463-476, 2001.

[6] Sun Shu-Jing, Liu Yu-Chen, Zhu Hu, "The dependence of Quorum Sensing in Serratia marcescens $\mathrm{JG}$ on The transcription of luxS gene", ARCH. MICROBIAL. 197 (5), 715$721,2015$.

[7] De Keersmacker, Sigrid C. J., Sonck Kathleen, Vanderleyden Jos, "Let luxS speak up in Al-2 Signalling", TRENDS in Microbiology. 14 (3), 114-119, 2006.

[8] Hussain Mahdi, Alammar Muheel, "Molecular Study of Some Virulence Factors Encoding genes of Enterobacter spp. Isolated from Different Clinical Specimens", Magazin of AL-kufa University for Biology. 5 (2), 1-13, 2013.

[9] Chen Guozhu, "Studies on the Control of gene Expression of $r p o S$ and its Regulon in E. Coli', $\mathrm{PhD}$, thesis, McMaster University, Hamilton, Ontario, 2003.

[10] Martinez-Garcia Esteban, Tormo Antonio, Navarro-Liorens Juana Maria, "Further Studies on rpoS in Enterobacteria: Identification of rpoS in Enterobactor cloacae and Kluyeracryo crescens", ARCH MICROBIAL. 175, 395-404, 2001.

[11] Kotewicz Michael L., Li Baoguang, Levy Dan D., Leclerc J. Eugene, Shifflet Andrew W., Cebula Thomas A., "Evolution of Multi-gene Segments in The Muts-RpoS Intergenic Region Salmonella enteric Serovar Typhimurium LT2”, MICROBIOLOGY+. 148, 2531-2540, 2002.

[12] Dong Tao, Schellhorn Herb E., "Global Effect of rpoS on gene Expression in Pathogenic Escherichia Coli 0157:H7 Strain EDL 933", BMC GENOMICS. 10 (349), 1-17, 2009.

[13] Kazmierczak Mark J., Wiedmann Martin, Boor Kathryn J., "Alternative Sigma Factors a", MICROBIOL MOL BIOL R. 69 (4), 227-543, 2005.

[14] Hengge-Aronis Regine, "Signal Transduction and Regulatory Mechanisms Involved in Control of the Sigma (rpoS) Subunit of RNA Polymerase", Microbiology Reviews. 66 (3), 373-395, 2002.

[15] Abid Intidhaar Naeem, "Bacteriological and Molecular Study of Enterobactor spp. Isolated from Different Clinical Specimens", PhD, thesis, University of Kufa, Iraq, 2013.

[16] Navarro-Lioréns J. M., Martinez-Garcia, Esteban, Tormo Antonio, "Enterobacter cloacae rpos Promoter and gene Organization", ARCH MICROBIAL. 179, 33-41, 2002.

[17] Stoeble Daniel M., Dorman Charles J., "The effect of Mobile Element IS10 on Experimental Regulatory Evolution in Escherichia coli", MOL BIOL EVOL. 27 (9), 20105-20112, 2010. 